\title{
Dietary exposure to heterocyclic amines in high-temperature cooked meat and fish in Malaysia.
}

\begin{abstract}
The intake of heterocyclic amines is influenced by the amount and type of meat and fish ingested, frequency of consumption, cooking methods, cooking temperature, and duration of cooking. In this study, the dietary intake of heterocyclic amines in Malaysia and their main sources were investigated. Forty-two samples of meat and fish were analysed by highperformance liquid chromatography with photodiode array detector to determine the concentration of the six predominant heterocyclic amines, namely: 2-amino-3methylimidazo[4,5-f] quinoline (IQ), 2-amino-3,4-dimethylimidazo[4,5-f] quinoline(MeIQ), 2-amino-3,8-dimethylimidazo[4,5-f] quinoxaline (MeIQx), 2-amino-3,4,8trimethylimidazo[4,5-f] quinoxaline (4,8-DiMeIQx), 2-amino-3,7,8-trimethylimidazo[4,5-f] quinoxaline (7,8-DiMeIQx), and 2-amino-1-methyl-6-phenylimidazo[4,5-b]pyridine (PhIP). Dietary intake data were obtained using a food-frequency questionnaire when interviewing 600 Malaysian respondents. The level of total heterocyclic amines in food samples studies ranged from not detected to $38.7 \mathrm{ng} \mathrm{g}(-1)$. The average daily intake level of heterocyclic amine was $553.7 \mathrm{ng}$ per capita day(-1). The intake of PhIP was the highest, followed by MeIQx and MeIQ. The results reveal that fried and grilled chicken were the major dietary source of heterocyclic amines in Malaysia. However, the heterocyclic amine intake by the Malaysian population was lower than those reported from other regions.
\end{abstract}

Keyword: Exposure assessment; Heterocyclic amines; Cooked foods. 